\title{
Differential Diagnosis between Oral Metastasis of Renal Cell Carcinoma and Salivary Gland Cancer
}

\author{
Yoshihiro Morita *, Kana Kashima, Mao Suzuki, Hiroko Kinosada, Akari Teramoto, Yuka Matsumiya and \\ Narikazu Uzawa (D)
}

check for updates

Citation: Morita, Y.; Kashima, K.; Suzuki, M.; Kinosada, H.; Teramoto, A.; Matsumiya, Y.; Uzawa, N. Differential Diagnosis between Oral Metastasis of Renal Cell Carcinoma and Salivary Gland Cancer. Diagnostics 2021, 11, 506. https://doi.org/10.3390/diagnostics 11030506

Academic Editor:

Michelangelo Fiorentino

Received: 30 January 2021

Accepted: 8 March 2021

Published: 12 March 2021

Publisher's Note: MDPI stays neutral with regard to jurisdictional claims in published maps and institutional affiliations.

Copyright: (C) 2021 by the authors. Licensee MDPI, Basel, Switzerland. This article is an open access article distributed under the terms and conditions of the Creative Commons Attribution (CC BY) license (https:/ / creativecommons.org/licenses/by/ $4.0 /)$.
Department of Oral and Maxillofacial Surgery II, Graduate School of Dentistry, Osaka University, 1-8 Yamadaoka, Suita-shi, Osaka 565-0871, Japan; k-kashima@dent.osaka-u.ac.jp (K.K.); mmaommao0429@dent.osaka-u.ac.jp (M.S.); h-kinosada@dent.osaka-u.ac.jp (H.K.); a-teramoto@dent.osaka-u.ac.jp (A.T.); yuka_loves_tigers@dent.osaka-u.ac.jp (Y.M.); uzawa@dent.osaka-u.ac.jp (N.U.)

* Correspondence: yoshi197@dent.osaka-u.ac.jp; Tel.: +81-6-6879-2941

Abstract: Renal cell carcinoma, which has clear cells in 70\% of cases, has a high frequency of hematogenous distant metastases to lung, bone, liver, and other areas. Metastatic cancer accounts for 1 to 3\% of malignant tumors in the stomatognathic region, and the metastasis of renal cell carcinoma to the oral mucosal tissue, though extremely rare, does occur. In addition, clear cells have been observed in some salivary gland cancers in the oral cavity. Therefore, the differential diagnosis of metastatic renal cell carcinoma and salivary gland cancer is important. This review discusses the differential diagnosis between metastatic renal cell carcinoma and malignant tumors of the salivary gland.

Keywords: renal cell carcinoma; salivary gland cancer; clear cell; differential diagnosis

\section{Introduction}

The oral cavity is an extremely rare site of cancer metastasis. Metastases account for approximately only $1 \%$ of all malignant tumors in the oral cavity [1]. When primary tumors metastasize to the oral cavity, the most common primary sites are lung carcinoma in males and breast carcinoma in females, followed by renal cell carcinoma (RCC) [2]. In RCC cases, approximately one third of the cases develop metastases. Distant metastases are detected in approximately half of RCC metastases cases after the initial diagnosis. The most common sites of distant metastases from RCC are the lungs, bone, liver, adrenal glands, contralateral kidney, and brain [3]. Although clear cells, which are either epithelial or mesenchymal cells consisting of pale or clear cytoplasm that have a distinct nucleus, can be observed in both benign and malignant epithelial, mesenchymal, melanocytic, and hematopoietic tumors, they are relatively rare in the head and neck region [4]. Because they share similar histological features, it is difficult to make definitive diagnosis in many of these tumors when clear cells predominate [5]. Focal clear cell changes may appear secondarily, reflecting clonal evolution, or may become more extensive with tumor progression [4]. The diagnosis of clear cell tumors may be made challenging by these changes.

\section{Renal Cell Carcinoma}

Renal cell carcinoma (RCC) accounts for more than $90 \%$ of cases of malignant renal disease and is thus the most common form. The incidence of RCC is high worldwide; it is the sixth most common cancer in men and the 10th most common in women [6], with a male-female ratio of 1.5:1. The peak incidence of RCC occurs in patients aged between 60 and 70 years.

There are several recognized histologic variants of RCC. Clear cell RCC accounts for $75 \%$ of cases, papillary RCC accounts for 10 to $15 \%$, and chromophobe RCC accounts for $5 \%$. These three histologic variants represent $90 \%$ of all RCCs [7]. Several risk factors that 
favor RCC development have been reported; these include a high body mass index [8], urinary stones in male patients [9], type 2 diabetes mellitus in female patients [10], chronic liver and kidney diseases [11], and the long-term use of analgesics [12], as well as a number of environmental factors [13]. As RCCs are frequently found in the course of diagnostic investigations for other purposes, they have been increasingly diagnosed in recent years.

The prognosis for RCC varies because it depends on the anatomical, histological, clinical, and molecular features of the disease. Additionally, in the genomic features, $9 p$ loss appears a reliable and promising marker of patients with renal cell carcinoma associated with a worse prognosis [14]. Overall, the 5-year survival rate for RCC is $74 \%$, but is lower in patients with stage III locoregional disease (54\%). Moreover, the survival rate decreases to $8 \%$ in patients with metastatic disease [15].

\section{Classification of Clear Cell Tumors in the Oral Cavity}

Physiologically and pathologically, clear cells can be classified broadly. Pathological clear cells are encountered in a variety of tumors, and physiological clear cells, which can either be epithelial or mesenchymal in origin, include both odontogenic and nonodontogenic tissues. It can therefore be said that clear cell tumors constitute a heterogeneous group of lesions.

\subsection{Clear Cell Metastatic Tumors}

Carcinomas from the kidney, liver, large bowel, prostate, and thyroid can metastasize to the maxillofacial area and are known to have the potential for clear cell differentiation; RCC does so most frequently [5]. RCC metastatic lesions can sometimes mimic salivary gland and odontogenic tumors, but are characterized by hemorrhagic areas and the prominent sinusoidal vascular component [16]. The prognosis of patients with metastatic carcinomas tends to be poor.

Local or distant metastases are known to occur in about one third of patients, and recurrence is seen in approximately $25 \%$ of patients with localized renal disease who undergo nephrectomy. RCC metastatic lesions typically develop in the lungs, regional lymph nodes, liver, bones, and brain [17].

Although relatively rare, RCC metastatic lesions are sometimes found in the oral cavity, predominantly involving the tongue, followed by the gingiva and maxillary bones $[18,19]$.

\subsection{Clear Cell Salivary Gland Tumors}

Although clear cell salivary gland tumors, which constitute less than $1 \%$ of all primary salivary gland tumors [20], are often malignant in nature, they include two types of benign lesions: clear cell variants of oncocytoma and myoepithelioma. Tumors that consist exclusively of myoepithelial cells are designated as myoepitheliomas, with both benign and malignant variants containing clear cells, although the latter is less common. Most malignant myoepitheliomas are less monomorphic than benign myoepitheliomas [21]. Epithelial myoepithelial carcinoma derives from intercalated ducts with biphasic duct-like structures. In acinic cell carcinoma, clear cells tend to occupy only a small portion of the tumor, whereas clear cell mucoepidermoid carcinoma can be easily identified by an admixture of clear squamous, mucous, and intermediate cells [16]. In addition, hyalinizing clear cell carcinoma demonstrates tumor cell clusters separated by broad bands of hyalinized stroma that have been shown to undergo myxoid or hyaline degeneration [16].

Clear cell metastatic tumors should be distinguished from primary salivary tumors because of their diagnostic and therapeutic importance [4]. In terms of biological behavior, clear cell salivary gland tumors are considered low-grade malignancies. This is because, in spite of their benign appearance, clear cell salivary gland tumors are capable of locally infiltrative growth and destruction, as well as metastasis, and tend to be associated with a poor prognosis [20]. 


\subsection{Other Clear Cell Lesions in the Oral Cavity}

Odontogenic neoplasms with significant clear cell components are exceedingly rare [22]. Most of these odontogenic lesions are composed of areas with characteristic histological features, which helps to distinguish them from each other [23].

Surface epidermal and cutaneous adnexal tumors are frequently found in the facial skin. Squamous and basal cell carcinomas have both been observed to show clear cell variants, with $90 \%$ of such neoplasms being found in the skin of the head and neck. Although individual islands within these neoplasms tend to consist of solely clear cells, adjacent cells have been reported to exhibit the typical characteristics of either basal or squamous cell carcinoma [23].

The incidence of lipoma in the oral cavity is known to be about 1\% [24]. In addition, liposarcoma accounts for about 5.6 to $9 \%$ of cases in the head and neck area [23]. Histologically, lipomas are characterized by mature adipose tissue without cytologic atypia [24]. On the other hand, liposarcoma shows the presence of lipoblasts, cellular pleomorphism, vascular proliferation, and mitotic activity. The prognosis of liposarcoma of the oral cavity is generally good owing to the small size of these neoplasms and the predominance of both myxoid and well-differentiated types [23].

Malignant melanomas and melanocytic nevi are composed of cells with different morphological phenotypes. Balloon cell melanoma, a rare form of vertical proliferative melanoma, is characterized by the nodular growth of neoplastic balloon cells. These tumors, which are characterized by large cell nests and sheets with clear or finely vacuolated cytoplasm, have a similar prognosis to other types of melanoma. In the largest series recorded, $57.5 \%$ of patients died of metastatic disease from 2 months to 12 years after initial surgery [23]. The clear cell variant of chondrosarcoma is very rare, accounting for only about $2 \%$ of all chondrosarcomas, with only nine cases having been reported in the head and neck area. In cases involving the head and neck, the histological findings have been consistent with the general characteristics of this entity throughout the body, and represent a component of conventional chondrosarcoma. The clear cell variant of chondrosarcoma is a distinct low-grade sarcoma with potential for local recurrence or distant metastasis, with a recurrence rate of approximately $20 \%$ reported in several case series [25].

\section{Differential Diagnosis of Clear Cell Tumors in the Oral Cavity}

Differentiating among clear cell tumors histologically is difficult by conventional light microscopy alone. This is especially true when trying to distinguish RCC metastases from clear cell malignancies of the salivary glands. The differential diagnosis between malignant salivary gland tumors including clear cells and metastasis of RCC is made using special staining or immunohistochemical staining based on histological characteristics. The major components of salivary gland tumors, which are characterized by clear cell changes, are contributed by the myoepithelial cells [26]. Based on this, primary salivary clear cell neoplasias can be divided into those that diagnostically require evidence of myoepithelial differentiation (myoepithelioma or myoepithelial carcinoma and epithelial myoepithelial carcinoma) and those that do not. Clear cell variants of salivary gland tumors, such as clear cell carcinoma, acinic cell carcinoma, mucoepidermoid carcinoma, and oncocytoma, do not have myoepithelial differentiation. In this regard, calponin-based immunohistochemistry can be an important diagnostic tool [26]. A chart for the differential diagnosis of clear cell malignant tumors is proposed in Figure 1. Schmidt et al. mentioned that the clinical usefulness of fine-needle aspiration cytology for the diagnosis of salivary gland lesions is controversial in their systematic review paper [27]. 


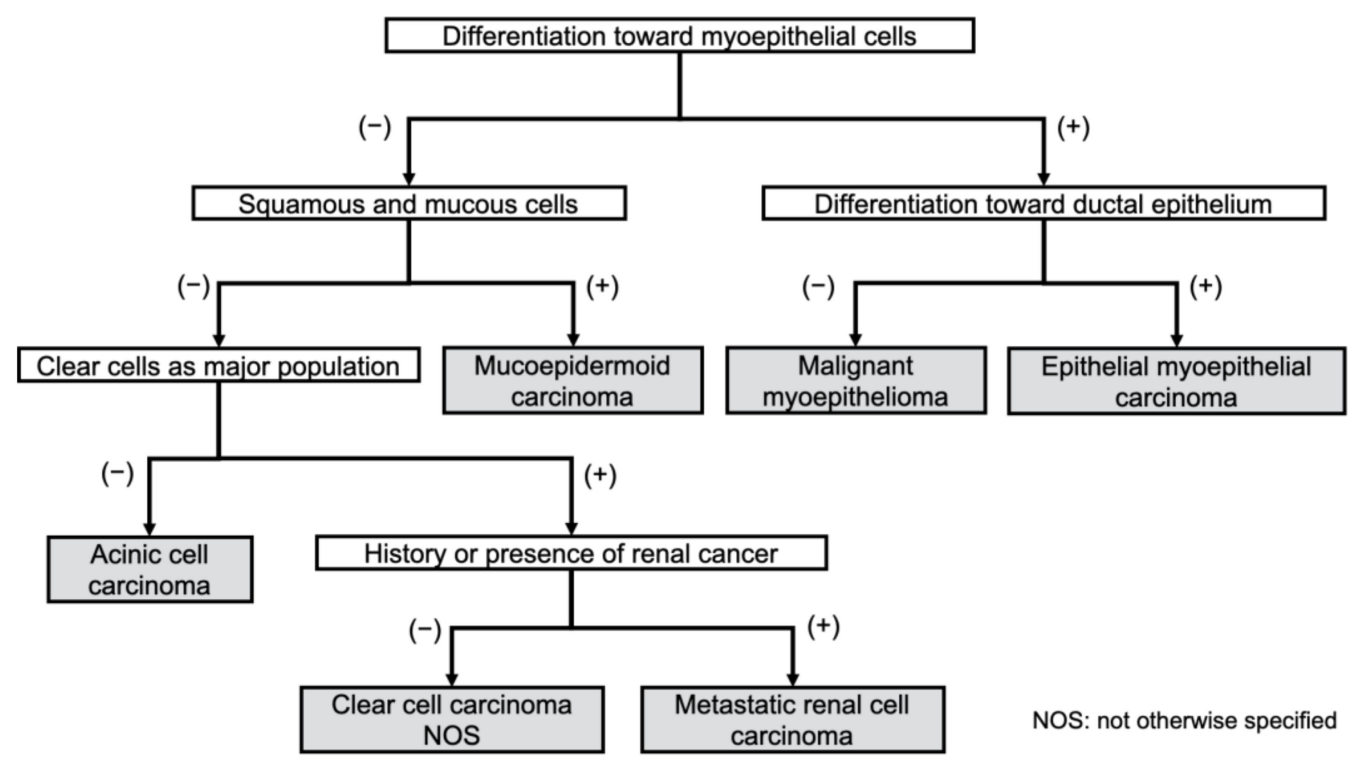

Figure 1. Diagnostic work chart of clear cell malignant tumors of the oral region.

\subsection{Characteristic Histopathological Findings for Making the Diagnosis}

Clear cell carcinomas of the salivary glands are usually seen as nests of clear cells divided by thin, fibrous connective septa and irregular vascular tissue. Most malignant myoepitheliomas frequently have high mitotic activity and atypical forms [28]. Table 1 shows the histological features useful for the differential diagnosis of salivary gland tumors containing malignant clear cells and metastatic RCC.

Table 1. Diagnostic immunohistochemical markers, special stains, and histopathological features for clear cell tumors.

\begin{tabular}{|c|c|c|c|}
\hline Name & Immunohistochemistry & Special Stains & Histopathology \\
\hline $\begin{array}{c}\text { Malignant } \\
\text { myoepitheliomas }\end{array}$ & $\begin{array}{c}\text { Myoepithelial } \\
\text { markers (+), p63(+) }\end{array}$ & $\begin{array}{c}\text { PAS }(+), \text { Mucin stains } \\
(-)\end{array}$ & - \\
\hline $\begin{array}{l}\text { Epithelial } \\
\text { myoepithelial } \\
\text { carcinoma }\end{array}$ & $\begin{array}{c}\text { Epithelial markers (+: } \\
\text { inner cells), } \\
\text { Myoepithelial } \\
\text { markers (+: outer } \\
\text { clear cells) }\end{array}$ & $\begin{array}{c}\text { PAS }(+), \text { Mucin stains } \\
(-)\end{array}$ & $\begin{array}{c}\text { Biphasic tubular } \\
\text { structure-outer clear } \\
\text { and inner cuboidal } \\
\text { cells }\end{array}$ \\
\hline $\begin{array}{l}\text { Mucoepidermoid } \\
\text { carcinoma }\end{array}$ & $\begin{array}{c}\text { Myoepithelial } \\
\text { markers (-), p63(+) }\end{array}$ & $\begin{array}{c}\text { PAS }(+), \text { Mucin stains } \\
(+)\end{array}$ & $\begin{array}{c}\text { Mucus and } \\
\text { intermediate cells }\end{array}$ \\
\hline Acinic cell carcinoma & Amylase (+) & $\operatorname{PAS}(+)^{*}$ & Granular cytoplasm \\
\hline Clear cell carcinoma & $\begin{array}{c}\text { Epithelial markers }(+), \\
\text { Myoepithelial } \\
\text { markers }(-)\end{array}$ & $\begin{array}{c}\text { PAS }(+), \text { Mucin stains } \\
(-)\end{array}$ & Hyalinizing stroma \\
\hline Renal cell carcinoma & $\begin{array}{l}\text { CD10(+), vimentin } \\
(+), \text { RCC marker }(+)\end{array}$ & $\begin{array}{l}\text { Oil red O stains }(+) \\
\text { Sudan black B stains } \\
(+)\end{array}$ & $\begin{array}{l}\text { Highly vascular } \\
\text { stroma, sinusoidal } \\
\text { spaces }\end{array}$ \\
\hline
\end{tabular}




\subsection{Special Stains for Making the Diagnosis}

\subsubsection{Periodic Acid-Schiff Stain}

Periodic acid-Schiff (PAS) stain is positive with glycogen, some mucins, and mucopolysaccharides, which can be diastase-sensitive or resistant. PAS diastase stain is a PAS stain used in combination with diastase, an enzyme that breaks down glycogen [26].

\subsubsection{Mucin Stains}

Neutral and acid-simple non-sulfated and acid-complex sulfated mucins and mucopolysaccharides are stained by a PAS stain, but not acid-simple mesenchymal mucins and acid-complex connective tissue mucins. Alcian blue is used to detect neutral and acidic mucopolysaccharides, sialomucin, and sulfomucin. Mucicarmine stains acidic mucin [26].

\subsubsection{Oil Red O and Sudan Black B Stains}

Oil red $\mathrm{O}$ and Sudan black B stain neutral lipids in frozen sections and lipoproteins in paraffin sections, and a combination of PAS with Alcian blue is a pan-mucin marker. Special stains used for the detection of glycogen and lipids in the cytoplasm of RCC cells are PAS, Oil red O, and Sudan black B [26].

\subsection{Immunohistochemical Stains for Diagnosis}

Immunohistochemical staining can assist in the diagnosis because RCC metastases show a strong reaction to vimentin, as well as focal cytokeratin (CK) positivity; by contrast, minor salivary gland cancers show diffuse CK positivity [12]. In addition, immunohistochemical staining for $\mathrm{p} 63$ has been shown to be useful for distinguishing mucoepidermoid carcinoma from some clear cell tumors [29], and Cluster of Differentiation (CD) 10 expression in RCCs are useful as a marker in the differential diagnosis of several tumors. Moreover, CD13 and GATA binding protein 3 (GATA-3) immunostains may serve as a diagnostic aid in differentiating subtypes of RCC [30].

\subsubsection{Myoepithelial Markers}

In addition to actin and S-100 [31], which are considered the classical myoepithelial markers, a number of myoepithelial markers are considered useful for immunohistochemical studies of formalin-fixed, paraffin-embedded tissues because they can recognize a variety of antigens in myoepithelial cells and have shown good sensitivity and specificity [31]. Among these myoepithelial markers, calponin, p63, CD10, and high-molecular weight cytokeratins (HMWCK; CK5/6 and CK14) have been confirmed to be effective in routine clinical practice because of their sensitivity, specificity, and ease of interpretation [31]. The utility of $\alpha$ smooth muscle actin ( $\alpha$-SMA), S-100, calponin, p63, CD10, CK5 /6, and CK14 for identifying the neoplastic myoepithelial components of adenomyoepithelioma has been reported by Moritani et al. [31], who concluded that all seven myoepithelial markers were useful for the diagnosis of adenomyoepithelioma. It is reasonable to select one or more markers from each group for the immunohistochemical panel of adenomyoepithelioma in consideration of the relatively high sensitivity of $\alpha$-SMA, calponin, and p63 in both spindle and clear cell lesions, as well as the unique paradoxical staining pattern and relatively high sensitivity of HMWCK in spindle cell lesions. When selecting immunohistochemical markers, taking the morphology of the lesion (i.e., spindle or clear cell) into account is also important.

Glial fibrillary acidic proteins (GFAPs) have also been reported to be sensitive markers of myoepithelial differentiation in salivary lesions [32].

\subsubsection{Epithelial Markers}

Both pancytokeratin (panCK) (AE1/AE3) and Epithelial membrane antigen (EMA) are epithelium-specific antibodies, and as the basic components of the cellular structure of normal epithelial and epithelial cancer cells, are frequently used to differentiate tumors in terms of whether they originate from the epithelium [33]. 


\subsubsection{Other Markers}

Although vimentin has commonly been used as a specific marker for neoplastic myoepithelial cells [34], its specificity has recently been reconsidered [35]. According to the results in this study, vimentin and calponin appear to be sensitive immunohistochemical markers of myoepithelial cells in salivary gland tumors, as they exhibit a predominantly intense expression. Therefore, the combination of calponin and vimentin is recommended for the identification of myoepithelial cells in neoplasms, as these cells can be found in various stages of differentiation [36].

Renal cell carcinoma marker (RCC-Ma) is a monoclonal antibody against a normal renal proximal tubule antigen. RCC-Ma expression is relatively specific for primary clear cell RCC [37]. Additionally, paired box 8 (PAX-8) and Carbonic anhydrase 9 (CA9, CAIX) expression are useful diagnostic markers for RCC $[38,39]$. PAX-8 expression is detected in the primary tumor and distant sites. Clear cell RCC has lower PAX-8 expression and is less frequently positive compared with normal tissue and other histological types. Therefore, the lack of expression does not exclude a tumor of renal origin. CA9 is not expressed in normal renal tissue but is expressed in most clear cell RCC through hypoxia inducible factor (HIF) $-1 \alpha$ accumulation driven.

\section{Review of the Literature for the Diagnosis of RCC Metastasis}

\subsection{Search Strategy}

A literature search was performed to retrieve previous studies describing the occurrence of oral metastases of renal cancer in the last 10 years. The following search items, combined with the Boolean term "AND," were used to perform an electronic search in the PubMed database: oral metastasis, renal cell carcinoma, case report. Only English ones were selected from the literatures obtained by this search.

\subsection{Review of the Literature}

In total, 65 articles published between 2001 and 2020 were found (Table 2); 49 (74.2\%) of the 66 cases reported were male (male-female ratio, 2.9:1), and patient ages ranged between 31 and 89 years (mean age, 52.6 years).

Table 2. Cases of metastases of renal cell carcinoma to the oral region: Review of the literature.

\begin{tabular}{|c|c|c|c|c|c|c|c|}
\hline $\begin{array}{l}\text { Metastasis } \\
\text { Site }\end{array}$ & Age (y) & Sex & $\begin{array}{c}\text { Time from } \\
\text { Diagnosis of RCC }\end{array}$ & Special Stains & IHC Markers & Year & Ref. \\
\hline Parotid gland & 64 & $\mathrm{M}$ & 10 years & Not performed & vimentin $(+)$, keratin $(+)$, CEA $(+)$ & 2001 & [40] \\
\hline Parotid gland & 61 & $\mathrm{M}$ & Same time & Not performed & Not performed & 2001 & [41] \\
\hline Parotid gland & 83 & $\mathrm{~F}$ & 10 years & Not performed & vimentin $(+)$, keratin $(+)$, CEA $(-)$ & 2002 & [42] \\
\hline Lip & 70 & $\mathrm{M}$ & Unknown & Not performed & vimentin $(+)$ & 2002 & [2] \\
\hline Tongue & 58 & $\mathrm{M}$ & Unknown & Not performed & Not performed & 2002 & [2] \\
\hline Tongue & 45 & $\mathrm{M}$ & 8 weeks & Not performed & Not performed & 2003 & [43] \\
\hline Tongue & 62 & $\mathrm{M}$ & Same time & Not performed & $\operatorname{AE} 1 / \operatorname{AE} 3(+), \operatorname{S100}(-), \operatorname{CEA}(-)$ & 2003 & [44] \\
\hline Tongue & 87 & $\mathrm{~F}$ & 10 years & PAS (+) & $\begin{array}{l}\text { CK }(+), \text { vimentin }(+), \operatorname{S100}(-), \\
\operatorname{MSA}(-), \operatorname{desmin}(-), \text { CEA }(-), \\
\text { HMB45 }(-), \text { CD31 }(-)\end{array}$ & 2004 & [12] \\
\hline Parotid gland & 59 & $\mathrm{~F}$ & 10 years & $\operatorname{PAS}(+)$ & $\begin{array}{l}\text { CK }(-), \text { vimentin }(+), \text { EMA }(+), \\
\text { CEA }(-), \text { SMA }(-), \text { S100 (-) }\end{array}$ & 2004 & [45] \\
\hline Parotid gland & 67 & $\mathrm{M}$ & Same time & Not performed & $\begin{array}{l}\text { LMWCK }(+), \text { vimentin }(+), \\
\text { CD10(+), S100(-), CK7 }(-), \\
\text { CK20(-), EMA }(-)\end{array}$ & 2005 & [5] \\
\hline Tongue & 49 & $\mathrm{~F}$ & 6 months & $\operatorname{PAS}(+)$ & Not performed & 2006 & [46] \\
\hline Parotid gland & 74 & $\mathrm{~F}$ & 7 years & Not performed & $\begin{array}{c}\text { panCK }(+), \operatorname{vimentin}(+), \text { S100 (-), } \\
\text { SMA }(-), \text { CK7 }(-), \text { CK20 (-) }\end{array}$ & 2007 & [47] \\
\hline
\end{tabular}


Table 2. Cont.

\begin{tabular}{|c|c|c|c|c|c|c|c|}
\hline $\begin{array}{l}\text { Metastasis } \\
\text { Site }\end{array}$ & Age (y) & Sex & $\begin{array}{c}\text { Time from } \\
\text { Diagnosis of RCC }\end{array}$ & Special Stains & IHC Markers & Year & Ref. \\
\hline Parotid gland & 58 & $\mathrm{~F}$ & Same time & Not performed & $\begin{array}{l}\text { CD10 (+), PNRA (+), vimentin }(+), \\
\text { AE1/AE3 (+), calponin }(-), \text { SMA } \\
(-), \text { S100 }(-), \text { CK7 }(-)\end{array}$ & 2008 & [48] \\
\hline Parotid gland & 76 & $\mathrm{~F}$ & 9 years & Not performed & $\begin{array}{c}\text { CD10 (+), PNRA }(+), \text { vimentin }(+), \\
\text { CK7 }(-)\end{array}$ & 2008 & [48] \\
\hline Parotid gland & 62 & $\mathrm{M}$ & 5 years & Not performed & $\begin{array}{l}\text { CAM5.2 (+), vimentin (+), CK18 } \\
(+), \text { CK8 (+), CK7 (-), PNRA (-) }\end{array}$ & 2008 & [48] \\
\hline Tongue & 78 & $\mathrm{M}$ & Same time & Not performed & Not performed & 2008 & [49] \\
\hline Tongue & 63 & $\mathrm{M}$ & 4 years & Not performed & $\begin{array}{c}\text { vimentin (+), CD10 (+), GCDFP } \\
(-), \text { S100 (-), HMB45 (-), MSA } \\
(-), \text { desmin }(-)\end{array}$ & 2008 & [3] \\
\hline Gingiva & 74 & $\mathrm{M}$ & Same time & Not performed & Not performed & 2008 & [50] \\
\hline Parotid gland & 69 & $\mathrm{M}$ & 1 year & Not performed & Not performed & 2008 & [51] \\
\hline Gingiva & 63 & $\mathrm{M}$ & 2 years & Not performed & Not performed & 2009 & [19] \\
\hline Gingiva & 52 & $\mathrm{M}$ & Same time & Not performed & Not performed & 2009 & {$[1]$} \\
\hline $\begin{array}{l}\text { Buccal } \\
\text { mucosa }\end{array}$ & 74 & $\mathrm{M}$ & 1 year & Not performed & Not performed & 2010 & [52] \\
\hline Tongue & 47 & $\mathrm{M}$ & Same time & Not performed & CD10 (+), EMA (+), AE1/AE3 (+) & 2011 & [53] \\
\hline Tongue & 48 & $\mathrm{~F}$ & 3 years & Not performed & Not performed & 2011 & [54] \\
\hline Gingiva & 63 & $\mathrm{M}$ & 2 years & Not performed & CD10 (+) & 2012 & [55] \\
\hline Tongue & 72 & $\mathrm{M}$ & Same time & Not performed & Not performed & 2012 & [56] \\
\hline $\begin{array}{l}\text { Submandibular } \\
\text { gland }\end{array}$ & 60 & $\mathrm{M}$ & 9 years & Not performed & Not performed & 2012 & [57] \\
\hline Tongue & 48 & $\mathrm{M}$ & 5 years & Not performed & Not performed & 2011 & [58] \\
\hline Parotid gland & 82 & $\mathrm{M}$ & 20 years & Not performed & Not performed & 2012 & [59] \\
\hline Lymph & 56 & $\mathrm{~F}$ & 6 years & Not performed & Not performed & 2011 & [60] \\
\hline Tongue & 64 & $\mathrm{~F}$ & 14 years & Not performed & $\begin{array}{c}\text { AE1/AE3 (+), EMA (+), vimentin } \\
(+), \text { CD10 (+), CK7 }(-), \text { CK19 (-), } \\
\text { CK20 (-), TTF1 (-) }\end{array}$ & 2012 & [61] \\
\hline Parotid gland & 79 & F & 16 years & Not performed & Not performed & 2012 & [62] \\
\hline Tongue & 70 & $\mathrm{M}$ & 16 years & Not performed & CD10 (+) & 2012 & [63] \\
\hline Tongue & 66 & $\mathrm{M}$ & Same time & Not performed & Not performed & 2013 & [64] \\
\hline Parotid gland & 44 & $\mathrm{~F}$ & 7 months & Not performed & Not performed & 2013 & [65] \\
\hline Tongue & 65 & $\mathrm{M}$ & 2 months & Not performed & Not performed & 2013 & [66] \\
\hline Tongue & 65 & $\mathrm{M}$ & Same time & Not performed & Not performed & 2013 & [66] \\
\hline $\begin{array}{l}\text { Mandible } \\
\text { bone }\end{array}$ & 57 & $\mathrm{M}$ & Same time & Not performed & $\begin{array}{c}\text { desmin }(+), \text { CK }(+), \text { vimentin }(+) \\
\text { S100 }(-), \operatorname{HMB} 45(-)\end{array}$ & 2013 & [67] \\
\hline Parotid gland & 64 & $\mathrm{M}$ & 6 years & $\operatorname{PAS}(+)$ & $\begin{array}{c}\text { AE1/AE3/CAM5.2 (+), vimentin } \\
(+), \text { RCC (+), CD10 (+), CA9 }(+), \\
\text { CK7 (-), CK20 (-), calponin }(-), \\
\text { p63 (-) }\end{array}$ & 2014 & [68] \\
\hline Tongue & 80 & $\mathrm{M}$ & 4 years & $\begin{array}{l}\text { Mucicarmine }(+) \\
\text { PAS }(+)\end{array}$ & CD10 (+), CK7 (-), Melan-A (-) & 2014 & [69] \\
\hline Lip & 64 & $\mathrm{M}$ & 6 months & Not performed & Not performed & 2014 & [70] \\
\hline Lip & 71 & $\mathrm{M}$ & 11 months & Not performed & $\begin{array}{c}\text { panCK }(+), \text { CD10 (+), vimentin } \\
(+), \text { CK7 }(-)\end{array}$ & 2014 & [71] \\
\hline
\end{tabular}


Table 2. Cont.

\begin{tabular}{|c|c|c|c|c|c|c|c|}
\hline $\begin{array}{l}\text { Metastasis } \\
\text { Site }\end{array}$ & Age (y) & Sex & $\begin{array}{c}\text { Time from } \\
\text { Diagnosis of RCC }\end{array}$ & Special Stains & IHC Markers & Year & Ref. \\
\hline Lip & 60 & $\mathrm{M}$ & 5 months & Not performed & Not performed & 2015 & [72] \\
\hline Tongue & 70 & $\mathrm{M}$ & Unknown & Not performed & $\begin{array}{l}\text { EMA (+), vimentin }(+), \operatorname{RCC}(+), \\
\text { CD10 (+), HMWCK }(+), \operatorname{CK7}(+)\end{array}$ & 2015 & [73] \\
\hline Gingiva & 51 & $\mathrm{M}$ & 3 years & Not performed & CD10 (+), PAX8 (+) & 2016 & [74] \\
\hline Gingiva & 60 & $\mathrm{M}$ & Same time & Not performed & Not performed & 2016 & [75] \\
\hline Gingiva & 31 & $\mathrm{~F}$ & 4 years & Not performed & $\begin{array}{c}\text { CD10 (+), vimentin }(+), \text { AE1 / AE3 } \\
(+)\end{array}$ & 2016 & [76] \\
\hline $\begin{array}{l}\text { Buccal } \\
\text { mucosa }\end{array}$ & 36 & $\mathrm{~F}$ & Same time & Not performed & $\begin{array}{c}\text { CD10 (+), PAX8(+), vimentin }(+) \\
\text { S100 (-) }\end{array}$ & 2016 & [77] \\
\hline Gingiva & 63 & $\mathrm{M}$ & Same time & Not performed & $\begin{array}{c}\text { vimentin (+), CD10 (+), CK (+), } \\
\text { PAX8 (+), CK5 (-), CK6 (-), S100 } \\
(-)\end{array}$ & 2017 & [78] \\
\hline Gingiva & 58 & $\mathrm{M}$ & Unknown & Not performed & vimentin (+), CA (+), CK7 (-) & 2017 & [79] \\
\hline Tongue & 55 & $\mathrm{M}$ & Same time & Not performed & $\begin{array}{l}\text { PAX8 (+), CD10 (+), AE1/AE3 (+), } \\
\text { CK7 (-), CK20 (-), TTF1 (-) }\end{array}$ & 2017 & [80] \\
\hline Maxilla bone & 54 & $\mathrm{M}$ & Same time & Not performed & CD10 (+) & 2018 & [81] \\
\hline Gingiva & 51 & M & Same time & Not performed & EMA $(+), \operatorname{Ki}-67(+), \operatorname{HMB} 45(-)$ & 2018 & [81] \\
\hline Gingiva & 78 & $\mathrm{~F}$ & Same time & Not performed & $\begin{array}{c}\text { panCK }(+), \text { CK8 } / 18(+), \text { PAX8 }(+), \\
\text { CD10 }(+), \text { CA9 }(+), \text { CK19 }(+), \\
\text { vimentin }(+), \text { EMA }(+), \text { CK20 }(-), \\
\text { CK7 }(-), \text { p63 }(-), \text { p40 }(-), \text { CK5 } \\
(-) \text {, synaptophysin }(-), \text { c-kit }(-), \\
\text { GATA3 }(-), \text { TTF1 }(-), \text { S100 }(-), \\
\text { CDX-2 (-), calponin }(-), \\
\text { calcitonin }(-), \text { EBER }(-), \text { HMB45 } \\
(-), \text { PR }(-), \text { ER }(-), \text { CD31 }(-)\end{array}$ & 2018 & [82] \\
\hline Tongue & 51 & $\mathrm{M}$ & Same time & Not performed & Not performed & 2018 & [18] \\
\hline Gingiva & 54 & $\mathrm{M}$ & 2 months & Not performed & $\begin{array}{c}\text { CD10 (+), vimentin }(+), \text { HMB45 } \\
(-), \text { S100 }(-)\end{array}$ & 2018 & [83] \\
\hline $\begin{array}{l}\text { Buccal } \\
\text { mucosa }\end{array}$ & 75 & $\mathrm{M}$ & 26 years & Not performed & $\begin{array}{c}\mathrm{CD} 10(+), \mathrm{SMA}(-), \mathrm{p} 63(-), \mathrm{S} 100 \\
(-)\end{array}$ & 2018 & [21] \\
\hline $\begin{array}{l}\text { Buccal } \\
\text { mucosa }\end{array}$ & 59 & $\mathrm{M}$ & Same time & Not performed & $\operatorname{PAX8}(+)$ & 2019 & [84] \\
\hline Tongue & 54 & $\mathrm{M}$ & Unknown & Not performed & Not performed & 2019 & [85] \\
\hline Parotid gland & 68 & $\mathrm{M}$ & Same time & Not performed & Not performed & 2019 & [86] \\
\hline Tongue & 61 & $\mathrm{M}$ & 6 months & Not performed & $\begin{array}{c}\text { vimentin }(+), \text { AE1/AE3 (+), } \\
\text { CAM5.2 (+), CD10 (+), EMA (+), } \\
\text { PAX8 (+), CK7 }(-), \text { CK20 (-), } \\
\text { CD117 (-), p40 (-) }\end{array}$ & 2020 & [87] \\
\hline $\begin{array}{l}\text { Buccal } \\
\text { mucosa }\end{array}$ & 71 & $\mathrm{M}$ & Same time & Not performed & $\begin{array}{c}\text { vimentin (+), AE1/AE3 (+), } \\
\text { CAM5.2 (+), CD10 (+), CK19 (+), } \\
\text { CK7 (-), CK20 (-), EMA (-), } \\
\text { CD117 (-), p40 (-) }\end{array}$ & 2020 & [87] \\
\hline $\begin{array}{l}\text { Mandible } \\
\text { bone }\end{array}$ & 56 & $\mathrm{~F}$ & Same time & Not performed & CD10 (+), PAX8 (+) & 2020 & [88] \\
\hline $\begin{array}{l}\text { Buccal } \\
\text { mucosa }\end{array}$ & 59 & $\mathrm{~F}$ & Same time & Not performed & $\begin{array}{c}\text { AE1 / AE3 }(+), \operatorname{S100~}(+), \operatorname{RCC}(+), \\
\text { Melan-A }(-), \operatorname{HMB} 45(-), \\
\text { myogenin }(-), \operatorname{chromogranin}(-), \\
\text { synaptophysin }(-)\end{array}$ & 2020 & [89] \\
\hline
\end{tabular}


Table 2. Cont.

\begin{tabular}{|c|c|c|c|c|c|c|c|}
\hline $\begin{array}{l}\text { Metastasis } \\
\text { Site }\end{array}$ & Age (y) & Sex & $\begin{array}{c}\text { Time from } \\
\text { Diagnosis of RCC }\end{array}$ & Special Stains & IHC Markers & Year & Ref. \\
\hline Gingiva & 89 & $\mathrm{M}$ & 7 years & PAS (+) & CD10 (+), AE1/AE3 (+) & 2020 & [90] \\
\hline Gingiva & 53 & $\mathrm{M}$ & Same time & Not performed & $\operatorname{RCC}(+)$ & 2020 & [91] \\
\hline
\end{tabular}

CEA: carcinoembryonic antigen, MSA: muscle-specific actin, HMB45: melanoma-associated antigen, LMWCK: low-molecular weight cytokeratins, PNRA: proximal nephron renal antigen, GCDFP: gross cystic disease fluid protein, TTF1: thyroid transcription factor-1, CA: carbonic anhydrase, PAX: Paired box protein, EBER: Epstein-Barr virus-encoded small RNAs, PR: progesterone receptor, ER: estrogen receptor.

A total of 35 of the 66 cases were previously diagnosed with RCC and had a history of treatment. The term from the initial diagnosis of RCC was up to 26 years, averaging 6.1 years. On the other hand, 26 cases were diagnosed with RCC in the kidney after the lesions in the oral cavity were found.

The tongue was the most common metastatic site in the oral region (33.3\%), followed by the parotid glands $(22.7 \%)$ and gingiva $(21.2 \%)$. Although only a few cases have been reported to have metastasized to bone $(4.5 \%)$, it is possible that some of the cases that metastasized to the gingiva included metastasized cases to the bone.

Immunohistochemical staining was performed in 39 cases for the diagnosis of metastatic lesions of RCC. It was suggested that immunohistochemical staining is not always necessary to diagnose RCC metastases, since the remaining 27 cases were diagnosed without immunohistochemistry. Among the cases that underwent immunohistochemical staining, vimentin, and CD10 were tested in many cases (61.4 and 64.1\%, respectively). In addition, the results of staining were positive in all of those cases. S-100 protein was tested in $35.9 \%$ of cases: one case was positive, and all others were negative. It appears that these immunohistochemical stains are useful for diagnosing RCC metastases.

\section{Conclusions}

It is known that RCC rarely metastasizes to the head and neck. Therefore, in patients with a history of RCC, metastatic RCC should be considered in the differential diagnosis of oral lesions. In addition, it should be considered that RCC may be found by whole body evaluation, such as positron emission tomography-computed tomography, even in patients with no history of RCC. In patients with a clear cell malignant tumor in the oral cavity, immunohistochemical staining is important to differentiate between metastatic RCC and malignant tumors of salivary gland origin.

Funding: This research received no external funding.

Institutional Review Board Statement: Not applicable.

Informed Consent Statement: Not applicable.

Data Availability Statement: No new data were created or analyzed in this study. Data sharing is not applicable to this article.

Conflicts of Interest: The authors declare no conflict of interest.

\section{References}

1. Maestre-Rodríguez, Ó.; González-García, R.; Mateo-Arias, J.; Moreno-García, C.; Serrano-Gil, H.; Villanueva-Alcojol, L.; Campos-De Orellana, A.M.; Monje-Gil, F. Metastasis of renal clear-cell carcinoma to the oral mucosa, an atypical location. Med. Oral Patol. Oral Cir. Bucal 2009, 14, e601-4. [CrossRef]

2. Pritchyk, K.M.; Schiff, B.A.; Newkirk, K.A.; Krowiak, E.; Deeb, Z.E. Metastatic renal cell carcinoma to the head and neck. Laryngoscope 2002, 112, 1598-1602. [CrossRef] [PubMed]

3. Will, T.A.; Agarwal, N.; Petruzzelli, G.J. Oral cavity metastasis of renal cell carcinoma: A case report. J. Med. Case Rep. 2008 , 2, 313. [CrossRef] 
4. Said-Al-Naief, N.; Klein, M.J. Clear cell entities of the head and neck: A selective review of clear cell tumors of the salivary glands. Head Neck Pathol. 2008, 2, 111-115. [CrossRef]

5. Seijas, B.P.; Franco, F.L.; Sastre, R.M.; García, A.Á.; Cembranos, J.L.L.C. Metastatic renal cell carcinoma presenting as a parotid tumor. Oral Surg. Oral Med. Oral Pathol. Oral Radiol. Endodontology 2005, 99, 554-557. [CrossRef]

6. Capitanio, U.; Bensalah, K.; Bex, A.; Boorjian, S.A.; Bray, F.; Coleman, J.; Gore, J.L.; Sun, M.; Wood, C.; Russo, P. Epidemiology of Renal Cell Carcinoma [Figure presented]. Eur. Urol. 2019, 75, 74-84. [CrossRef]

7. Lopez-Beltran, A.; Carrasco, J.C.; Cheng, L.; Scarpelli, M.; Kirkali, Z.; Montironi, R. 2009 update on the classification of renal epithelial tumors in adults. Int. J. Urol. 2009, 16, 432-443. [CrossRef]

8. MacLeod, L.C.; Hotaling, J.M.; Wright, J.L.; Davenport, M.T.; Gore, J.L.; Harper, J.; White, E. Risk factors for renal cell carcinoma in the VITAL study. J. Urol. 2013, 190, 1657-1661. [CrossRef]

9. Cheungpasitporn, W.; Thongprayoon, C.; O'corragain, O.A.; Edmonds, P.J.; Ungprasert, P.; Kittanamongkolchai, W.; Erickson, S.B. The risk of kidney cancer in patients with kidney stones: A systematic review and meta-analysis. QJM 2015, 108, 205-212. [CrossRef] [PubMed]

10. Joh, H.K.; Willett, W.C.; Cho, E. Type 2 diabetes and the risk of renal cell cancer in women. Diabetes Care 2011, $34,1552-1556$. [CrossRef] [PubMed]

11. Christensson, A.; Savage, C.; Sjoberg, D.D.; Cronin, A.M.; Frank O’Brien, M.; Lowrance, W.; Nilsson, P.M.; Vickers, A.J.; Russo, P.; Lilja, H. Association of cancer with moderately impaired renal function at baseline in a large, representative, population-based cohort followed for up to 30 years. Int. J. Cancer 2013, 133, 1452-1458. [CrossRef]

12. Marioni, G.; Gaio, E.; Poletti, A.; Derosas, F.; Staffieri, A. Uncommon metastatic site of renal adenocarcinoma: The oral tongue. Acta Otolaryngol. 2004, 124, 197-201. [CrossRef] [PubMed]

13. Kabat, G.C.; Navarro Silvera, S.A.; Miller, A.B.; Rohan, T.E. A cohort study of reproductive and hormonal factors and renal cell cancer risk in women. Br. J. Cancer 2007, 96, 845-849. [CrossRef] [PubMed]

14. Di Nunno, V.; Mollica, V.; Brunelli, M.; Gatto, L.; Schiavina, R.; Fiorentino, M.; Santoni, M.; Montironi, R.; Caliò, A.; Eccher, A.; et al. A Meta-Analysis Evaluating Clinical Outcomes of Patients with Renal Cell Carcinoma Harboring Chromosome 9P Loss. Mol. Diagn. Ther. 2019, 23, 569-577. [CrossRef]

15. Kidney and Renal Pelvis Cancer-Cancer Stat Facts. Available online: https://seer.cancer.gov/statfacts/html/kidrp.html (accessed on 2 January 2021).

16. Maiorano, E.; Altini, M.; Favia, G. Clear cell tumors of the salivary glands, jaws, and oral mucosa. Semin. Diagn. Pathol. 1997, 14, 203-212. [PubMed]

17. McKay, R.R.; Kroeger, N.; Xie, W.; Lee, J.L.; Knox, J.J.; Bjarnason, G.A.; MacKenzie, M.J.; Wood, L.; Srinivas, S.; Vaishampayan, U.N.; et al. Impact of bone and liver metastases on patients with renal cell carcinoma treated with targeted therapy. Eur. Urol. 2014, 65, 577-584. [CrossRef] [PubMed]

18. Đanić, P.; Đanić, D.; Macan, D. Tongue metastasis as an initial presentation of renal cell carcinoma. Med. Glas. 2018, 15, 52-58. [CrossRef]

19. Makos, C.P.; Psomaderis, K. A Literature Review in Renal Carcinoma Metastasis to the Oral Mucosa and a New Report of an Epulis-Like Metastasis. J. Oral Maxillofac. Surg. 2009, 67, 653-660. [CrossRef]

20. Nichols, C.; Geist, R.Y.; Nichols, K.M. Clear cell neoplasm. J. Laryngol. Otol. 1992, 106, 553-555. [CrossRef] [PubMed]

21. Morita, Y.; Iwagami, T.; Kawakita, C.; Kusuyama, Y.; Niki-Yonekawa, A.; Morita, N. Oral metastasis of renal cell carcinoma mimicking recurrence of excised malignant myoepithelioma: A case report. Mol. Clin. Oncol. 2018, 9, 66. [CrossRef] [PubMed]

22. Benton, D.C.; Eisenberg, E. Clear cell odontogenic carcinoma: Report of a case. J. Oral Maxillofac. Surg. 2001, 59, 83-88. [CrossRef] [PubMed]

23. Premalatha, B.; Neethi, H. Clear Cell Tumors of the Head and Neck: An Overview. World J. Dent. 2012, 3, 344-349. [CrossRef]

24. Cakarer, S.; Selvi, F.; Isler, S.C.; Soluk, M.; Olgac, V.; Keskin, C. Intraosseous lipoma of the mandible: A case report and review of the literature. Int. J. Oral Maxillofac. Surg. 2009, 38, 900-902. [CrossRef]

25. Mokhtari, S.; Mirafsharieh, A. Clear cell chondrosarcoma of the head and neck. Head Neck Oncol. 2012, 4, 1-5. [CrossRef] [PubMed]

26. Kar, A.; Pattnaik, K.; Kar, T.; Biswal, P.; Mishra, C.; Guru, L. Clear cell lesions in pathology: Histomorphologic approach to diagnosis. Indian J. Pathol. Microbiol. 2020, 63, 177. [CrossRef] [PubMed]

27. Schmidt, R.L.; Hall, B.J.; Wilson, A.R.; Layfield, L.J. A Systematic Review and Meta-Analysis of the Diagnostic Accuracy of Fine-Needle Aspiration Cytology for Parotid Gland Lesions. Am. J. Clin. Pathol. 2011, 136, 45-59. [CrossRef] [PubMed]

28. Richa; Ray, J.G.; Mohanty, S.P. Vibha Malignant myoepithelioma of palate. Contemp. Clin. Dent. 2012, 3, 370-372. [CrossRef]

29. O'Sullivan-Mejia, E.D.; Massey, H.D.; Faquin, W.C.; Powers, C.N. Hyalinizing clear cell carcinoma: Report of eight cases and a review of literature. Head Neck Pathol. 2009, 3, 179-185. [CrossRef]

30. Brunelli, M.; Erdini, F.; Cima, L.; Eccher, A.; Fioravanzo, A.; Gobbo, S.; Segala, D.; Ghimenton, C.; Mazzoleni, G.; Munari, E.; et al. Proximal CD13 Versus Distal GATA-3 Expression in Renal Neoplasia According to WHO 2016 Classification. Appl. Immunohistochem. Mol. Morphol. 2018, 26, 316-323. [CrossRef] [PubMed] 
31. Moritani, S.; Ichihara, S.; Yatabe, Y.; Hasegawa, M.; Iwakoshi, A.; Hosoda, W.; Narita, M.; Nagai, Y.; Asai, M.; Ujihira, N.; et al. Immunohistochemical expression of myoepithelial markers in adenomyoepithelioma of the breast: A unique paradoxical staining pattern of high-molecular weight cytokeratins. Virchows Arch. 2015, 466, 191-198. [CrossRef]

32. Hornick, J.L.; Fletcher, C.D.M. Myoepithelial tumors of soft tissue: A clinicopathologic and immunohistochemical study of 101 cases with evaluation of prognostic parameters. Am. J. Surg. Pathol. 2003, 27, 1183-1196. [CrossRef]

33. Yang, Y.; Li, J.; Mao, S.; Zhu, H. Comparison of immunohistology using pan-CK and EMA in the diagnosis of lymph node metastasis of gastric cancer, particularly micrometastasis and isolated tumor cells. Oncol. Lett. 2013, 5, 768-772. [CrossRef]

34. De Araujo, V.C.; de Araujo, N.S. Vimentin as a marker of myoepithelial cells in salivary gland tumors. Eur. Arch. Oto Rhino Laryngol. 2004, 247, 252-255. [CrossRef] [PubMed]

35. De Araújo, V.C.; de Sousa, S.O.M.; Carvalho, Y.R.; de Araújo, N.S. Application of Immunohistochemistry to the Diagnosis of Salivary Gland Tumors. Appl. Immunohistochem. Mol. Morphol. 2000, 8, 195-202. [CrossRef]

36. Cavalcante, R.B.; Lopes, F.F.; Ferreira, A.S.; de Almeida Freitas, R.; de Souza, L.B. Immunohistochemical expression of vimentin, calponin and HHF-35 in salivary gland tumors. Braz. Dent. J. 2007, 18, 192-197. [CrossRef]

37. Perna, A.G.; Ostler, D.A.; Ivan, D.; Lazar, A.J.F.; Diwan, A.H.; Prieto, V.G.; Reed, J.A. Renal cell carcinoma marker (RCC-Ma) is specific for cutaneous metastasis of renal cell carcinoma. J. Cutan. Pathol. 2007, 34, 381-385. [CrossRef] [PubMed]

38. Tostain, J.; Li, G.; Gentil-Perret, A.; Gigante, M. Carbonic anhydrase 9 in clear cell renal cell carcinoma: A marker for diagnosis, prognosis and treatment. Eur. J. Cancer 2010, 46, 3141-3148. [CrossRef]

39. Barr, M.L.; Jilaveanu, L.B.; Camp, R.L.; Adeniran, A.J.; Kluger, H.M.; Shuch, B. PAX-8 expression in renal tumours and distant sites: A useful marker of primary and metastatic renal cell carcinoma? J. Clin. Pathol. 2015, 68, 12-17. [CrossRef] [PubMed]

40. Li, L.; Friedrich, R.E.; Schmelzle, R.; Donath, K. Metachronous bilateral metastases of renal cell carcinoma to the parotid region. J. Oral Maxillofac. Surg. 2001, 59, 434-438. [CrossRef] [PubMed]

41. Kundu, S.; Eynon-Lewis, N.J.; Radcliffe, G.J. Extensive metastatic renal cell carcinoma presenting as facial nerve palsy. J. Laryngol. Otol. 2001, 115, 488-490. [CrossRef] [PubMed]

42. Park, Y.W.; Hlivko, T.J. Teaching case: Parotid gland metastasis from renal cell carcinoma. Laryngoscope 2002, 112, 453-456. [CrossRef]

43. Lang, E.E.; Patil, N.; Walsh, R.M.; Leader, M.; Walsh, M.A. A case of renal cell carcinoma metastatic to the nose and tongyue. Ear Nose Throat J. 2003, 82, 382-383. [CrossRef] [PubMed]

44. Goel, M.C.; Williams, D.W.; Evans, H.; Roberts, J.G. Lingual metastasis from renal cell carcinoma management and review of the literature. Urol. Int. 2003, 71, 418-421. [CrossRef] [PubMed]

45. Göğüş, Ç.; Kiliç, Ö.; Tulunay, Ö.; Tulunay, Ö.; Bedük, Y. Solitary metastasis of renal cell carcinoma to the parotid gland 10 years after radical nephrectomy. Int. J. Urol. 2004, 11, 894-896. [CrossRef] [PubMed]

46. Torres-Carranza, E.; Garcia-Perla, A.; Infante-Cossio, P.; Belmonte-Caro, R.; Loizaga-Iriondo, J.-M.M.; Gutierrez-Perez, J.-L.L. Airway obstruction due to metastatic renal cell carcinoma to the tongue. Oral Surg. Oral Med. Oral Pathol. Oral Radiol. Endod. 2006, 101, e76-e78. [CrossRef] [PubMed]

47. Newton, J.R.; O'Donnell, M.; Samuel, P.R. A case of renal cell carcinoma metastasizing to the parotid gland. Otolaryngol. Head Neck Surg. 2007, 136, S65-S67. [CrossRef] [PubMed]

48. Mrena, R.; Leivo, I.; Passador-Santos, F.; Hagström, J.; Mäkitie, A.A. Histopathological findings in parotid gland metastases from renal cell carcinoma. Eur. Arch. Oto Rhino Laryngol. 2008, 265, 1005-1009. [CrossRef]

49. Azam, F.; Abubakerr, M.; Gollins, S. Tongue metastasis as an initial presentation of renal cell carcinoma: A case report and literature review. J. Med. Case Rep. 2008, 2, 249. [CrossRef]

50. Matamala, G.N.; De los Ángeles Fernández Toro, M.; Ugarte, E.V.; Mendoza, M.L.; Alcayaga, G.R. Oral metastasis of renal cell carcinoma, presentation of a case. Med. Oral Patol. Oral Cir. Bucal 2008, 13, E742-E744.

51. Spreafico, R.; Nicoletti, G.; Ferrario, F.; Scanziani, R.; Grasso, M. Metastasi parotidea da carcinoma a cellule renali: Caso clinico e revisione della letteratura. Acta Otorhinolaryngol. Ital. 2008, 28, 266-268.

52. Zhang, Y.; Gu, Z.Y.; Tian, Z.; Yang, C.; Cai, X.Y. Oral metastasis from primary transitional cell carcinoma of the renal pelvis: Report of a case. Int. J. Oral Maxillofac. Surg. 2010, 39, 737-739. [CrossRef]

53. Yoshitomi, I.; Kawasaki, G.; Mizuno, A.; Nishikido, M.; Hayashi, T.; Fujita, S.; Ikeda, T. Lingual metastasis as an initial presentation of renal cell carcinoma. Med. Oncol. 2011, 28, 1389-1394. [CrossRef]

54. Morvan, J.B.; Veyrires, J.B.; Mimouni, O.; Cathelinaud, O.; Allali, L.; Verdalle, P. Clear-cell renal carcinoma metastasis to the base of the tongue and sphenoid sinus: Two very rare atypical ENT locations. Eur. Ann. Otorhinolaryngol. Head Neck Dis. 2011, 128, 91-94. [CrossRef]

55. Schwab, B.; Lee, W.T. Bilateral renal cell carcinoma metastasis in the oral cavity. Am. J. Otolaryngol. Head Neck Med. Surg. 2012, 33, 154-155. [CrossRef] [PubMed]

56. Balliram, S.; Goetz, L.; Ramsoobhag, K.; Narinesingh, D.; Medford, S.; Naraynsingh, V. Renal cell carcinoma presenting as a tongue lesion. J. Oral Maxillofac. Surg. 2012, 70, 1605-1608. [CrossRef] [PubMed]

57. Serouya, S.M.; Dultz, L.A.; Concors, S.J.; Wang, B.; Patel, K.N. Late solitary metastasis of renal cell carcinoma to the submandibular gland. J. Oral Maxillofac. Surg. 2012, 70, 2356-2359. [CrossRef] [PubMed] 
58. Wadasadawala, T.; Kumar, P.; Agarwal, J.; Ghosh-Laskar, S. Palliation of dysphagia with radiotherapy for exophytic base tongue metastases in a case of renal cell carcinoma. Indian J. Urol. 2011, 27, 550-552. [CrossRef]

59. Deeb, R.; Zhang, Z.; Ghanem, T. Metastatic renal cell carcinoma to the parotid gland in the setting of chronic lymphocytic leukemia. Case Rep. Med. 2012, 2012, 265708. [CrossRef]

60. Özkiriş, M.; Kubilay, U.; Sezen, O. Cervical lymph node metastasis in renal cell carcinoma. J. Oral Maxillofac. Pathol. 2011, 15, 211-213. [CrossRef]

61. Ghazali, N.; Davis, C.; Barrett, A.W.; Tighe, J.V. Bilateral Asynchronous Renal Cell Carcinoma with Metastatic Involvement of the Tongue. Case Rep. Pathol. 2012, 2012, 1-4. [CrossRef] [PubMed]

62. Lau, S.Y.C.; Chittleborough, T.J.; Mccracken, J.A.; Wijeratne, S. Metastatic clear-cell renal carcinoma to the parotid. ANZ J. Surg. 2012, 82, 760-761. [CrossRef]

63. Porta, C.; Ganini, C.; Lasagna, A.; Ferraris, E.; Gatti, P.; Paglino, C.; Imarisio, I.; Morbini, P.; Benazzo, M. Lingual metastasis from renal cell carcinoma: A case report and literature review. Rare Tumors 2012, 4, 130-133. [CrossRef]

64. Mazeron, R.; Fenoll, L.; Mathieu, M.C.; Dumas, I.; Haie-Meder, C. Brachytherapy for isolated tongue metastasis of renal clear cell carcinoma. Eur. Ann. Otorhinolaryngol. Head Neck Dis. 2013, 130, 149-151. [CrossRef] [PubMed]

65. Yanlan, C.; Liping, S.; Shaomin, C.; Zi, L. Metastasis to the parotid region as an initial presentation of renal cell carcinoma: A case report. Oncol. Lett. 2013, 5, 997-999. [CrossRef]

66. Ray, A.; Bhattacharya, J.; Ganguly, S. Renal cell carcinoma presenting with oral tongue metastasis: A rare case presentation. J. Cancer Res. Ther. 2013, 9, 117-118. [CrossRef] [PubMed]

67. Ahmadnia, H.; Amirmajdi, N.M.; Mansourian, E. Renal cell carcinoma presenting as mandibular metastasis. Saudi J. Kidney Dis. Transpl. 2013, 24, 789-792. [CrossRef]

68. Udager, A.M.; Rungta, S.A. Metastatic renal cell carcinoma, clear cell type, of the parotid gland: A case report, review of literature, and proposed algorithmic approach to salivary gland clear cell neoplasms in Fine-needle aspiration biopsies. Diagn. Cytopathol. 2014, 42, 974-983. [CrossRef]

69. Abbaszadeh-Bidokhty, H.; Motallebnejad, M.; Rajabi-Moghaddam, M. Metastatic Renal Cell Carcinoma Presenting as a Clear-Cell Tumor in Tongue: A Case Report; Mashhad University of Medical Sciences: Mashhad, Iran, 2014; Volume 26.

70. Kotak, A.; Merrick, G. Presentation of metastatic renal cell carcinoma as a lip lesion. J. Surg. Case Rep. 2014, 2014, rju083. [CrossRef]

71. Suojanen, J.; Färkkilä, E.; Helkamaa, T.; Loimu, V.; Törnwall, J.; Lindqvist, C.; Hagström, J.; Mesimäki, K. Rapidly growing and ulcerating metastatic renal cell carcinoma of the lower lip: A case report and review of the literature. Oncol. Lett. 2014, 8, 2175-2178. [CrossRef] [PubMed]

72. Jatti, D.; Puri, G.; Aravinda, K.; Dheer, D.S. An atypical metastasis of renal clear cell carcinoma to the upper lip: A case report. J. Oral Maxillofac. Surg. 2015, 73, 371.e1-371.e6. [CrossRef]

73. Altuntaş, O.; Petekkaya, I.; Süslü, N.; Güllü, I. Renal cell carcinoma metastatic to the tongue: A case report and review of the literature. J. Oral Maxillofac. Surg. 2015, 73, 1227-1230. [CrossRef]

74. Selvi, F.; Faquin, W.C.; Michaelson, M.D.; August, M. Three synchronous atypical metastases of clear cell renal carcinoma to the maxillary gingiva, scalp and the distal phalanx of the fifth digit: A case report. J. Oral Maxillofac. Surg. 1286, 74, 1286.e1-1286.e9. [CrossRef] [PubMed]

75. Ali, R.A.E.; Mohamed, K.E.H. Metastatic clear cell renal cell carcinoma presenting with a gingival metastasis. Clin. Pract. 2016, 6, 24-26. [CrossRef]

76. Guimarães, D.M.; Pontes, F.S.C.; Miyahara, L.A.N.; Guerreiro, M.Y.R.; De Almeida, M.C.L.; Pontes, H.A.R.; Dos Santos Pinto, D. Metastatic renal cell carcinoma to the oral cavity. J. Craniofac. Surg. 2016, 27, e533-e534. [CrossRef]

77. Kudva, R.; Nayal, B.; Kantipudi, S.; Ray, S. Metastatic renal cell carcinoma of the buccal mucosa masquerading as a salivary gland neoplasm. J. Oral Maxillofac. Pathol. 2016, 20, 547. [CrossRef] [PubMed]

78. Georgy, J.T.; Mathuram, A.J.; George, A.A.; Chandramohan, J. Renal cell carcinoma presenting as a cutaneous horn and nodules on the gingiva and scalp. BMJ Case Rep. 2017, 2017, bcr2017. [CrossRef]

79. Nifosì, G.; Bressand, H.; Nifosì, A.F.; Nifosì, L.; Damseaux, P. Epulis-Like Presentation of Gingival Renal Cancer Metastasis. Case Rep. Oncol. 2017, 10, 758-763. [CrossRef]

80. Raiss, H.; Duplomb, S.; Tartas, S.; Layachi, M.; Errihani, H. Lingual metastasis as an initial presentation of renal cell carcinoma: A case report. J. Med. Case Rep. 2017, 11, 1-7. [CrossRef]

81. Derakhshan, S.; Rahrotaban, S.; Mahdavi, N.; Mirjalili, F. Metastatic renal cell carcinoma presenting as maxillary lesion: Report of two rare cases. J. Oral Maxillofac. Pathol. 2018, 22, S39-S43. [CrossRef]

82. Vasilyeva, D.; Peters, S.; Philipone, E.; Yoon, A.J. Renal cell carcinoma metastatic to the maxillary gingiva: A case report and review of the literature. J. Oral Maxillofac. Pathol. 2018, 22, S102-S107. [PubMed]

83. Kishore, M.; Chauhan, D.S.; Dogra, S. Unusual presentation of renal cell carcinoma: A rare case report. J. Lab. Physicians 2018, 10, 241-244. [CrossRef]

84. Nesbitt, A.L.; Lim, Z.L.T.; Chan, K.J.; Zardawi, I.; Pridgeon, S.W. Metastatic renal cell carcinoma presenting with both acute stroke and an oral lesion. Urol. Case Rep. 2019, 23, 75-77. [CrossRef] 
85. Abro, C.; Sedhom, R.; Soni, A.; Markowski, M. Cutaneous finger and tongue metastases in renal cell carcinoma. BMJ Case Rep. 2019, 12, e230516. [CrossRef] [PubMed]

86. Netto, R.; de Freitas Filho, S.A.J.; Cortezzi, W.; Merly, F.; de Andrade, V.M.; Pires, F.R. Metastasis of Renal Cell Carcinoma Causing Significant Facial Asymmetry. Case Rep. Surg. 2019, 2019, 1-5. [CrossRef] [PubMed]

87. Nisi, M.; Izzetti, R.; Graziani, F.; Gabriele, M. Renal Cell Carcinoma Metastases to the Oral Cavity: Report of 2 Cases and Review of Literature. J. Oral Maxillofac. Surg. 2020, 78, 1557-1571. [CrossRef] [PubMed]

88. Zhang, R.; Lee, C.W.; Basyuni, S.; Santhanam, V. Mandibular swelling as the initial presentation for renal cell carcinoma: A case report. Int. J. Surg. Case Rep. 2020, 70, 96-100. [CrossRef] [PubMed]

89. Patel, S.; Barros, J.; Nwizu, N.N.; Ogbureke, K.U.E. Metastatic renal cell carcinoma to the oral cavity as first sign of disease: A case report. Clin. Case Rep. 2020, 8, 1517-1521. [CrossRef]

90. Nishii, N.; Shimamoto, H.; Ohsako, T.; Yokokawa, M.; Sato, Y.; Ohata, Y.; Kayamori, K.; Ikeda, T.; Harada, H. Renal cell carcinoma metastasis to the maxillary bone successfully treated with surgery after vascular embolization: A case report. J. Med. Case Rep. 2020, 14, 1-7. [CrossRef]

91. Stojanovic, M.; Krasic, D.; Trajkovic, M.; Petrovic, V. Rare renal cell carcinoma metastasis to mandibular gingiva: A case report and literature review. Niger. J. Clin. Pract. 2020, 23, 1483-1486. [CrossRef] [PubMed] 